\title{
Linking social context and addiction neuroscience: a computational psychiatry approach
}

\author{
Andrea Reiter, Andreas Heinz and Lorenz Deserno
}

In their recent article (Time to connect: bringing social context into addiction neuroscience (Nat. Rev. Neurosci. 17, 592-599 (2016)) ${ }^{1}$, Heilig et al. suggest that the lack of progress towards treatment and prevention of addiction is partly due to a neglect of social factors in neuroscientific research of addiction. We share the authors' disappointment but argue that merely broadening the focus towards social context is not sufficient to close the "large gap [that] exists between the promise of neuroscientific approaches to addiction and what they have delivered". To date, we lack sufficient transfer of powerful theoretical accounts and methodological resources to understand the multiple facets of addiction. Thus, adding yet another obviously important component to the heterogeneity that characterizes addiction might be insufficient to make addiction neuroscience more clinically relevant. Rather, a conceptual shift in 'addiction neuroscience' may be warranted.

Seminal epidemiological and neuroimaging studies have established associations between neurobiological measures, addiction and social status ${ }^{2-4}$, thereby generating hypotheses on the physiological, psychological and social components of addiction. In our view, the next step should be to determine how different neural computations in modulatory circuits give rise to certain psychological phenomena of learning and decision making that are associated with addictive behaviours, such as cue-induced drug craving and habitual drug intake despite negative consequences. To integrate these interacting physiological, behavioural and social levels of description ${ }^{5}$, a generative model of addictive behaviours is needed. To achieve this goal, we propose that coherent theoretical accounts be formalized and applied in stringent and translational experimental design. A developmental perspective is necessary to track the onset, maintenance and relapse of addiction in longitudinal studies. Computational models might prove valuable for any of these processes.
Computational models aid theory building by formalizing hypotheses and rigorously capture relations between latent factors and observations. They can inform on particularly meaningful manipulations and enable mechanistic interpretation of experimental results. Indeed, addictive behaviours and social cognition can be tied together through existing theoretical accounts of addictive behaviours - for example, those based on reinforcement learning ${ }^{6}$ and the 'Bayesian brain' hypothesis ${ }^{7}$. Computational modelling has begun to inform empirical studies on decision making in addiction ${ }^{8,9}$, putatively addiction-like disorders $^{9,10}$ and risk factors for addiction ${ }^{11-13}$. Likewise, computational accounts of social cognition have been tested in neuroscience. Research combining computational modelling of social behaviours with neuroimaging suggests that social information may be processed by mechanisms similar to those involved in (non-social) reward-based learning and decision making ${ }^{14}$. Thus, establishing a mechanistic theory of addictive behaviours should advance empirical knowledge of whether (or not) social adversities are indeed specific factors that contribute to certain aspects of addictive behaviours. Ultimately, this might allow one to define patient-specific combinations of various model parameters and model evidences for alternative (social and non-social) disease mechanisms ${ }^{15}$. Crucially, such quantifiable 'computational fingerprints' have to be examined rigorously regarding their predictive power in longitudinal studies that include children or teenagers before the onset of addiction. This might prompt targeted intervention and prevention - be it in the social or the nonsocial domain - informed by the mechanisms that give rise to addiction.

Andrea Reiter is at the Department for Lifespan Developmental Neuroscience, Technical University Dresden, 01062 Dresden, Germany; and at the Department of Neurology, Max Planck Institute for Human Cognitive and Brain Sciences, 04103 Leipzig, Germany.
Andreas Heinz is at the Department of Psychiatry and Psychotherapy, Charité Universitätsmedizin Berlin, 10117 Berlin, Germany.

Lorenz Deserno is at the Department of Child and Adolescent Psychiatry, Psychotherapy and Psychosomatics, University of Leipzig, 04103 Leipzig, Germany; and at the Department of Neurology, Max Planck Institute for Human Cognitive and Brain Sciences, 04103 Leipzig, Germany.

Correspondence to L.D. deserno@cbs.mpg.de

doi: $10.1038 / \mathrm{nrn} .2017 .67$ Published online 19 Jun 2017

1. Heilig, M., Epstein, D. H., Nader, M. A. \& Shaham, Y. Time to connect: bringing social context into addiction neuroscience. Nat. Rev. Neurosci. 17, 592-599 (2016).

2. Morgan, D. et al. Social dominance in monkeys: dopamine $D_{2}$ receptors and cocaine selfadministration. Nat. Neurosci. 5, 169-174 (2002).

3. Martinez, D. et al. Dopamine type 2/3 receptor availability in the striatum and social status in human volunteers. Biol. Psychiatry 67, 275-278 (2010).

4. Heinz, A. J., Beck, A., Meyer-Lindenberg, A. Sterzer, P. \& Heinz, A. Cognitive and neurobiological mechanisms of alcohol-related aggression. Nat. Rev. Neurosci. 12, 400-413 (2011).

5. Huys, Q. J., Maia, T. V. \& Frank, M. J. Computational psychiatry as a bridge from neuroscience to clinical applications. Nat. Neurosci. 19, 404-413 (2016).

6. Dayan, P. Dopamine, reinforcement learning, and addiction. Pharmacopsychiatry 42 (Suppl. 1), S56-S65 (2009)

7. Schwartenbeck, P. et al. Optimal inference with suboptimal models: addiction and active Bayesian inference. Med. Hypotheses 84, 109-117 (2015).

8. Reiter, A. M. F. et al. Behavioral and neural signatures of reduced updating of alternative options in alcohol-dependent patients during flexible decision-making. J. Neurosci. 36, 10935-10948 (2016).

9. Voon, V. et al. Disorders of compulsivity: a common bias towards learning habits. Mol. Psychiatry 20 345-352 (2015).

10. Reiter, A. M., Heinze, H. J., Schlagenhauf, F. \& Deserno, L. Impaired flexible reward-based decisionmaking in binge eating disorder: evidence from computational modeling and functional neuroimaging. Neuropsychopharmacology 42, 628-637 (2017).

11. Reiter, A. M., Deserno, L., Wilbertz, T., Heinze, H. J. \& Schlagenhauf, F. Risk factors for addiction and their association with model-based behavioral control. Front. Behav. Neurosci. 10, 26 (2016).

12. Deserno, L. et al. Lateral prefrontal model-based signatures are reduced in healthy individuals with high trait impulsivity. Transl Psychiatry 5, e659 (2015).

13. Otto, A. R., Raio, C. M., Chiang, A., Phelps, E. A. \& Daw, N. D. Working-memory capacity protects modelbased learning from stress. Proc. Natl Acad. Sci. USA 110, 20941-20946 (2013).

14. Behrens, T. E., Hunt, L. T., Woolrich, M. W. \& Rushworth, M. F. Associative learning of social value. Nature 456, 245-249 (2008).

15. Stephan, K. E., Iglesias, S., Heinzle, J. \& Diaconescu, A. O. Translational perspectives for computational neuroimaging. Neuron $\mathbf{8 7}, 716-732$ (2015).

\section{Acknowledgements}

This work was in part supported by the German Research Foundation (DFG FOR 1617, DFG SFB 940/2 B7).

\section{Competing interests statement}

The authors declare no competing interests. 